\title{
¿LÍDER PEDAGÓGICO O GERENTE DE ESCUELA? EVOLUCIÓN DEL ROL DEL DIRECTOR DE ESCUELA EN CHILE
}

\author{
Fabián Campos $^{1}$, René Valdés ${ }^{2}$ y Paula Ascorra ${ }^{3}$
}

\begin{abstract}
RESUMEN
En la búsqueda de la mejora de la educación, el Ministerio de Educación de Chile (Mineduc) ha impulsado durante los últimos años una serie de reformas que directa o indirectamente afectan el trabajo de los directores. A la luz de los principios del nuevo management público (NMP), y utilizando un análisis documental, el presente artículo estudia la evolución que ha tenido el rol de director en las políticas públicas de los últimos 40 años. Los resultados revelan: 1) que la totalidad de los elementos característicos del NMP (trabajo individual, competencia, orientación a cumplir indicadores y planes de corto plazo) están presentes en las políticas, lo que configura un rol gerencial del director de escuela; 2) que durante los últimos tres años se han dictado políticas alejadas de los principios del NMP, las que proponen un nuevo rol para el director: el del líder pedagógico; 3) que no se pasa evolutivamente de un rol a otro, sino que ambos conviven en la escuela, generando una posible tensión que el director debe resolver. Se discute la real factibilidad que tienen los directores de ejercer su rol de líder pedagógico, el cual está orientado a la construcción colaborativa de confianzas y capacidades en la escuela, en un escenario marcado por una larga historia de políticas que propician el trabajo individual y con indicadores a cumplir en el corto plazo, principios que son propios del NMP y que responden más adecuadamente al trabajo de un gerente de empresa.
\end{abstract}

Conceptos clave: accountability, director de escuela, liderazgo, neoliberalismo, nuevo management público, políticas públicas, rendición de cuentas.

\section{INSTRUCTIONAL LEADER OR SCHOOL MANAGER? THE EVOLUTION OF THE PRINCIPAL'S ROLE IN CHILE}

\begin{abstract}
In the quest to improve education, the Ministry of Education of Chile (Mineduc) has promoted during the last years, a series of reforms that directly or indirectly affect the work of school principals. In light of the principles of the New Public Management (NPM) and, using a documentary analysis, this article studies the evolution of the role of director in the public policies of the last 40 years. The results reveal: 1) that all the characteristic elements of the NPM (individual work, competence, orientation to meet indicators, short-term plans) are present in the policies, which implicate a managerial role of the school director. 2) That during the past
\end{abstract}

1 Pontificia Universidad Católica de Valparaíso, Valparaíso, Chile. Contacto: fabian.campos@pucv.cl

2 Pontificia Universidad Católica de Valparaíso, Valparaíso, Chile. Contacto: rene.valdes@pucv.cl

3 PontificiaUniversidad Católica de Valparaíso, Valparaíso, Chile. Contacto: paula.ascorra@pucv.cl 
3 years, policies have been dictated away from the principles of the NPM that generate a new role for the director, that of the instructional leader. 3) That one role does not evolve into the other, but rather both coexist in schools, generating a possible tension that the director must resolve. The real feasibility of school principals to exercise their role as an instructional leader oriented to the collaborative construction of trust and abilities in the school is discussed, in a scenario marked by a long history of policies that favor individual work with indicators that must be met in the short term, principles that are specific to the NPM, and are more related to the work of a company manager.

Key concepts: accountability, leadership, neoliberalism, new public management, public policies, school principal.

\section{Introducción}

Durante los últimos años, el Ministerio de Educación de Chile (Mineduc) ha impulsado una serie de reformas que directa o indirectamente afectan el trabajo de los directores de establecimientos educacionales (Nuñez, Weinstein y Muñoz, 2012). Una influencia importante para estas políticas proviene del nuevo management público (en adelante NMP). Este movimiento global nació en la década de los ochenta con el objeto de incrementar la eficiencia y eficacia en la gestión de negocios. Los parámetros diseñados por el NMP para empresas privadas rápidamente se transfirieron a las políticas de Estado (Van der Sluis, Reezigt \& Borghans, 2015). Conceptos como fiscalización, rendición de cuentas, cumplimiento de indicadores y competencia se fueron instalando - poco a poco- en los diseños de políticas educativas, de modo que implementar una nueva lógica de gestión en el ámbito educativo demandó nuevos roles de los agentes con mayor responsabilidad, es decir, los directores de escuela.

Estudiar la evolución del rol directivo en Chile es particularmente relevante porque se trata del primer país donde se instalaron cabalmente los principios del NMP a inicios de los ochenta, lo que lo convirtió en el gran experimento en educación (Bellei, 2015; Redondo, 2005), donde era esperable observar un cambio en el rol de los directores. Por otra parte, la historia política de Chile, que incluye una dictadura (1973-1989), gobiernos de centro derecha (1990-1999), gobiernos social demócratas (2000-2009; 2014-2017) y dos gobiernos de derecha (2010-2013; 2017-2021) puede otorgar 
una panorámica acerca de la influencia del NMP en las políticas educacionales con independencia del sector político.

Este estudio adhiere al llamado que realiza Ball (1993) a los investigadores de política educativa, motivándolos a "reconocer y analizar la existencia de discursos 'dominantes' como el neoliberalismo y la teoría del management dentro de la política social" (p. 15). Mediante el análisis documental de un conjunto de leyes y normativas en educación, y a la luz de los principios del NMP, se analiza la evolución del rol del director de escuela durante los últimos 40 años.

Este estudio busca contribuir a la discusión de la política pública en educación de Chile, la que ha centrado parte de la mejora educativa en los roles y funciones que ejercen los directores. Develar el tipo de rol que la política pública les demanda puede contribuir a explicitar las limitaciones y posibilidades que estos agentes educativos enfrentan a la hora de favorecer la transformación educacional.

\section{La lógica del NMP y su impacto en la educación}

En la década de 1970 comenzó a bosquejarse una nueva etapa en la historia del mundo occidental. Las crisis energéticas (o del petróleo) de 1973 y 1979 pusieron en jaque no solo a las economías mundiales, sino que fueron el catalizador de una transformación tan profunda, que implicó dejar atrás el mundo como lo conocíamos. No solo cambió la empresa privada, que levantó un nuevo conjunto de reglas para sobrevivir y ser competitiva, sino también la administración pública. Uno de los efectos fue la crítica que se hizo al modelo de bienestar, ya que los Estados no eran capaces de sostener el gasto público, situación que gatilló la aparición de nuevas reglas y el surgimiento del NMP.

El NMP es un cuerpo filosófico de ideas de gestión que se transmiten desde la esfera privada al mundo público con el propósito de hacerlo más efectivo y eficiente (Verger \& Curran, 2014). Uno de los preceptos guía del NMP es que los gobiernos deberían limitarse a ocupar un rol mínimo en la provisión de servicio públicos, reduciendo el aparato y el gasto del Estado (World Bank, 1997). Las 
principales características del modelo (Gewirtz \& Ball, 2000; Hood, 1991; Pollitt, 1995; Verger y Normand, 2015; Wittmann, 2008) son:

- Favorecer los instrumentos y los estilos de gestión del sector privado, es decir, propiciar la profesionalización.

- Instalar normas y medidas de desempeño explícitas.

- Instalar indicadores de resultados más que de procesos.

- Desagregar y descentralizar los servicios públicos, entendiendo el proceso como transformación de las organizaciones burocráticas tradicionales en agencias independientes.

- Hacer hincapié en la competencia como un incentivo para el logro.

- Introducir contratos a plazo fijo, pago en función del rendimiento y la determinación local de sueldos y condiciones laborales.

- Reducción de costos, limitación de los presupuestos y mayor transparencia en la asignación de recursos.

El Banco Mundial y el Fondo Monetario Internacional fueron los encargados de propiciar la instalación de este modelo en el ámbito de la educación en los países en vías de desarrollo (Mundy \& Menashy, 2014; Verger \& Curran, 2014). Fruto de ello, la adscripción a los principios de NMP se convirtió en un fenómeno global (Verger \& Curran, 2014) que comenzó en los años ochenta como parte de los ajustes estructurales que exigía el Banco Mundial, a cambio de importantes préstamos en dinero para levantar las alicaídas economías locales (Bonal, 2002; Hall et al., 2015). Hoy en día, esta institución se mantiene como la principal fuente de financiamiento internacional, con un presupuesto de miles de millones de dólares para reformas en educación (Mundy \& Verger, 2015).

Como toda política que adquiere el título de "global", el paquete asociado al NMP fue mutando durante su travesía por el mundo, y no necesariamente llegó completo a todos los territorios, ya que su implementación fue negociada en función de los factores contextuales propios de cada lugar (Verger y Normand, 2015). Por ejemplo, un fuerte liderazgo político y sistemas de rendición 
de cuentas claros, hicieron la diferencia entre Singapur ${ }^{4}$ y Malasia (éxito en la implementación del NMP), versus Bangladesh y Sri Lanka (fracaso) (Samaratunge, Alam \& Teicher, 2008). Los factores propios del Estado de Quebec llevaron a una recontextualización del NMP en una lógica más neoestatista que neoliberal, con una mayor centralización y regulación de la burocracia clásica (Maroy, Mathou, Vaillancourt \& Voisin, 2015). Van der Sluis, Reezigt y Borghans (2015) revisaron para el caso holandés la tensión que experimentaban las escuelas producto de la convivencia de políticas inspiradas en los principios del NMP con otras de corte local, que contradecían en parte esos principios. Brasil presentó las particularidades propias de la implementación del NMP en un gobierno federal, a lo que se sumó el rol de una sociedad civil empoderada que durante los últimos años ha tensionado a los denominados "gobiernos populares democráticos" (Andrade Oliveira, 2015). Factores económicos y políticos son los responsables de una instalación parcial del NMP en Cataluña, que a su vez está llena de contradicciones y omisiones (Verger y Curran, 2015). La tradición republicana, un gremio de profesores poderoso y padres que no se involucran en este debate son los elementos que han impedido que el NMP se instale en el sistema educativo francés, a diferencia de lo ocurrido en otras áreas en el mismo país, como es el caso de la salud (Derouet, Normand y Pacheco, 2015). En Noruega, si bien desde inicios de los ochenta que se abordaron estas reformas, no fue hasta principios del nuevo siglo — momento en que este país fue catalogado como uno de los peores en rendimiento en la prueba PISA y en otras evaluaciones internacionales- cuando estas políticas se aceleraron y profundizaron (Møller \& Skedsmo, 2013). La transformación en la relación Estado-escuela, la creación de cuasi mercados en el campo de la educación y la privatización de la educación pública son algunos de los efectos producidos en Italia (Grimaldi, Serpieri \& Taglietti, 2015). En Inglaterra el NMP implicó cambios no solo en términos de estructuras e incentivos, sino también en la instalación de un conjunto de nuevos valores y de un ambiente moral que se reflejó en una reorientación de los fines originales de la escuela, dejando lejos su primer énfasis en las necesidades inmediatas de su comunidad, por el añorado "mejoramiento" a través de la

4 Ver Aoki (2015) para una revisión más acabada del caso de Singapur. 
búsqueda de "clientes" altamente valorados por sobre los necesitados (Gewirtz \& Ball, 2000) .

Haciendo una analogía con un iceberg, podemos comprender mejor el impacto que ha tenido la implementación de estos cambios estructurales en el mundo. Lo que es rápidamente observable en la superficie del iceberg es la descentralización de la educación, su marketización, el cambio en su financiamiento, la instalación de evaluaciones estandarizadas que permiten medir desempeño (tanto de estudiantes como de profesores), y la rendición de cuentas internas y externas (Falabella, 2014; Mandiola \& Ascorra, 2010); pero por debajo de esto, queda mucho por investigar.

Chile no ha estado ajeno a la neoliberalización de las políticas educativas. Según Lerena y Trejos (2015), las consecuencias del modelo neoliberal en Chile han sido claras y sus resultados han hecho más dificultoso el camino hacia una educación de calidad. Algunos de los efectos clave que destacan los autores son:

- El paso de un sujeto comunitario a un individuo inserto en una sociedad diferenciada;

- la implicancia del voucher ${ }^{6}$ ha gatillado la competitividad entre instituciones escolares, donde cada estudiante es un "valor de cambio";

- la incorporación de la rendición de cuentas ha generado presión y sobrecarga de los actores escolares; y

- debido a la desprofesionalización docente, los profesores no participan de la deliberación de la enseñanza (enfoques pedagógicos y didácticos). Esto se traduce en "enseñar para responder pruebas", afectando la diversificación de la enseñanza y flexibilidad curricular.

5 Otra mirada de la instalación del NMP en estos últimos tres países (Noruega, Italia e Inglaterra) puede revisarse en Hall et al. (2015).

6 El voucher o "bono educativo" fue una propuesta realizada en los años cincuenta por el economista Milton Friedman para producir un mercado competitivo de sistemas educativos. En Chile el voucher funciona a través de lo que se conoce como Estado subsidiario; esto significa que el Estado no garantiza el derecho a la educación, sino que financia (subsidia) el acceso a esta. Este subsidio se materializa en un bono educativo (voucher) que recibe el sostenedor (encargado distrital de educación) en función de la asistencia media de los estudiantes. 
Si bien esto ha ocurrido en materia de educación en general, es necesario profundizar de manera más exclusiva en la evolución del rol del director a la luz de los principios del NMP.

\section{Políticas dirigidas a directores de escuelas y liceos en Chile: ¿Qué sabemos del rol de los directores hasta ahora?}

Las políticas son enunciados operacionales de los valores de la sociedad (Bell \& Stevenson, 2006; Kogan, 1975; Ranson, 1995). De esta manera, van construyendo "verdades" que permiten transitar hacia esos valores ideales, excluyendo aquellas declaraciones que caracterizan como falsas y manteniendo en circulación aquellas que se esgrimen como verdaderas (Ball, Maguire, Braun \& Hoskins, 2011). La política proporciona así un lenguaje y un marco de acción para pensar, hablar y actuar en torno a lo que sucede en la escuela (Tseng, 2015). En ese sentido, se ha descrito la performatividad de las políticas, pues ellas no solo determinan lo que se debe o no hacer, sino que construyen roles, posiciones y subjetividades (Hall \& McGinity, 2015; Montecinos, Pino, Campos-Martínez, Domínguez $\&$ Carreño, 2014; Sisto, 2012). En estas transformaciones, el rol que ejerce el director juega un papel clave para la articulación discursiva de la política, la creación de significados y la acción al interior de la escuela (Ball, Maguire, Braun \& Hoskins, 2011).

Estudios internacionales han identificado al director como el segundo factor intraescuela que mayor impacto tiene en los aprendizajes de los estudiantes (Gurr-Mark, Drysdale-George \& Mulford, 2010; Leithwood, Day, Sammons, Harris \& Hopkins, 2006). Por otra parte, investigaciones nacionales revelan que el liderazgo del director y su equipo es el principal factor en las trayectorias de mejora escolar (Bellei, Valenzuela, Vanni y Contreras, 2014), lo cual es coincidente con investigaciones que relevan las acciones de liderazgo del director como predictivas del clima y de la mejora escolar (Bulach, Boothe \& Pickett, 2006; López-Alfaro, Osorio-González, GallegosAraya y Cáceres-Cadena, 2016). Esta contundente evidencia es la que ha impulsado políticas educativas especialmente dirigidas a 
directores: formación, selección, marcos de actuación y rendición de cuentas, las que se revisan a continuación.

En los años noventa, el director de escuela era considerado poco relevante en los procesos de enseñanza y aprendizaje. Su labor en el marco del estatuto docente se focalizaba en la "dirección, administración, supervisión y coordinación de la educación" (Ley $\mathrm{N}^{\circ} 19.070$, art. $7^{\circ}$ ). En el año 2004 , se otorgaron mayores atribuciones a los directores, concretamente, se los hizo participar de estrategias curriculares, desarrollo profesional docente y relación con padres y apoderados (Nuñez et al., 2012). El 2005 se difundió en Chile el Marco para la buena dirección, documento elaborado por el Mineduc que precisa un conjunto de dimensiones y acciones que deseablemente debían ejercer los directores escolares (Mineduc, 2005). Cuatro años después, con la conformación de la Ley General de Educación (Ley $\mathrm{N}^{\circ} 20.370$ ) se consagra (discursivamente) un nuevo rol para el director que se denomina "líder pedagógico", centrado en la conducción del proyecto educativo y en elevar el desempeño escolar de los alumnos.

El año 2011, mediante la Ley $N^{0} 20.501$, se establecieron nuevos lineamientos para la selección de directores escolares. En ese año se reforzó la idea de que estos eran actores relevantes en la creación de equipos de trabajo. Luego, en 2015, surgió el Nuevo marco para la buena dirección y liderazgo escolar, documento que vino a actualizar y reemplazar el de 2005 (Mineduc, 2015). Este instrumento intenta dar una respuesta comprensiva respecto de las prácticas de liderazgo, entendiéndolo como una acción situada y compartida y, por lo tanto, no dependiente solo del director. Esta vez, el marco se orienta al ejercicio de un liderazgo pedagógico, presentando cinco dimensiones de prácticas:

- construyendo e implementando una visión estratégica compartida,

- desarrollando las capacidades profesionales,

- liderando y monitoreando los procesos de enseñanza y aprendizaje,

- gestionando la convivencia y la participación de la comunidad escolar,

- desarrollando y gestionando la organización. 
Muchas de estas políticas dirigidas a directores van generando cargas de trabajo que van más allá de las definiciones de liderazgo. Se trata de acciones implícitas en el cargo, que no figuran en la descripción formal que se hace de él, pero que las investigaciones han revelado que sí existen. La evidencia levantada hasta ahora concuerda en que los directores de escuela se han convertido en portadores locales del NMP, responsables por la implementación de las distintas reformas educativas que llevan su espíritu (aplicación de pruebas estandarizadas a estudiantes, evaluaciones de profesores, cumplimiento de indicadores para la obtención de recursos, etc.) (Hall et al., 2015; Montecinos, Ahumada, Galdames, Campos \& Leiva, 2015; Sisto y Fardella, 2011). Ahora bien, es preciso conocer cuál o cuáles son los roles del director que dichas políticas han configurado durante los últimos 40 años, si este ha evolucionado o no, y si existe consistencia longitudinal en el levantamiento de ese rol, a la luz de los distintos gobiernos y énfasis educacionales que ha tenido cada uno.

\section{Método}

El diseño del estudio es de tipo documental (Peña y Pirela, 2007). Para la producción de información, se utilizó la técnica de recopilación documental. Esta tiene como finalidad recopilar datos e información a partir de fuentes documentales (Montero y Hochman, 2005). Según Saavedra y Farías (2014), y Stecher (2010), el mensaje que emite la política no es relevante solo por su significado, sino también por las intencionalidades que subyacen a este. En ese sentido, las leyes son portadoras de concepciones básicas, aspectos éticos y formas de comprensión del mundo. Dicho esto, se analizaron 20 documentos clave de la política educativa chilena (Tabla 1). Los criterios establecidos para seleccionar estos fueron los siguientes:

- que fueran propositivos, es decir, que orientaran a los directores a desarrollar acciones y tomar decisiones;

- que fueran representativos de todos los gobiernos de los últimos 40 años (dictadura, gobierno de centroderecha, gobierno social demócrata y gobierno de derecha); y

- que tuvieran como principal objetivo mejorar la calidad de la educación chilena. 
Tabla 1

Corpus textual por año y tipo

\begin{tabular}{|c|c|c|}
\hline Año & Tipo & Documento \\
\hline 1980 & Decreto Ley & $\begin{array}{l}\text { No } 3.476 \text { Sobre Subvenciones a Establecimientos de Enseñanza } \\
\text { Particular Subvencionados por el Estado. }\end{array}$ \\
\hline 1980 & Decreto & $\begin{array}{l}\text { No } 8.144 \text { Reglamenta Decreto Ley No } 3.476 \text {, de 1980, sobre } \\
\text { Subvenciones a Establecimientos Particulares Gratuitos de } \\
\text { Enseñanza }\end{array}$ \\
\hline 1981 & $\begin{array}{l}\text { Decreto con } \\
\text { Fuerza de } \\
\text { Ley }\end{array}$ & $\begin{array}{l}\text { No 1-3.063 Reglamenta Aplicación Inciso Segundo del Artículo } \\
38 \text { del DL. No 3.063, de } 1979 \text { (municipalización). }\end{array}$ \\
\hline 1995 & Ley & $\begin{array}{l}\text { No } 19.410 \text { Creación del Sistema Nacional de Evaluación de } \\
\text { Desempeño. }\end{array}$ \\
\hline 1998 & $\begin{array}{l}\text { Decreto con } \\
\text { Fuerza de } \\
\text { Ley }\end{array}$ & $\begin{array}{l}\text { No } 2 \text { Fija Texto Refundido, Coordinado y Sistematizado del DFL } \\
\text { No } 2 \text {, de 1996, sobre Subvención del Estado a Establecimientos } \\
\text { Educacionales. }\end{array}$ \\
\hline 2004 & Ley & No 19.961 Evaluación Docente. \\
\hline 2004 & Ley & No 19.979 Modificación Jornada Escolar Completa. \\
\hline 2005 & Documento & Marco para la buena dirección (MBD). \\
\hline 2006 & Ley & $\begin{array}{l}\text { No } 20.006 \text { Concursabilidad Directores Establecimientos } \\
\text { Municipales. }\end{array}$ \\
\hline 2006 & Decreto & $\begin{array}{l}N^{\circ} 177 \text { Modifica Decreto No } 453 \text {, de 1991(Estatuto de los } \\
\text { Profesionales de la Educación). }\end{array}$ \\
\hline 2008 & Ley & No 20.248 Subvención Escolar Preferencial (SEP). \\
\hline 2009 & Ley & No 20.370 Ley General de Educación. \\
\hline 2011 & Ley & No 20.501 Calidad y Equidad de la Educación. \\
\hline 2011 & Ley & $\begin{array}{l}\text { No } 20.529 \text { Sistema Aseguramiento de la Calidad de la } \\
\text { Educación. }\end{array}$ \\
\hline 2011 & Ley & No 20.550 Modifica Ley No 20.248 SEP. \\
\hline 2012 & Ley & $\begin{array}{l}\text { No } 20.567 \text { Modifica Ley No } 20.248 \text { de Subvenciones Escolares, } \\
\text { en Materia de Rendición. }\end{array}$ \\
\hline 2014 & Documento & $\begin{array}{l}\text { Estándares indicativos de desempeño para establecimientos } \\
\text { educacionales y sus sostenedoresl. }\end{array}$ \\
\hline 2015 & Documento & Marco para la buena dirección y liderazgo escolar (MBDLE). \\
\hline 2016 & Ley & $\begin{array}{l}\text { No } 20.903 \text { Crea el Sistema de Desarrollo Profesional Docente y } \\
\text { Modifica otras Normas. }\end{array}$ \\
\hline 2017 & Ley & No 21.040 Crea el Sistema de Educación Pública. \\
\hline
\end{tabular}

Fuente: Elaboración propia.

1 Estos estándares fueron desarrollados por la Agencia de Calidad de la Educación, ACE, al amparo de la Ley $N^{\circ}$ 20.529. La ACE es una de las cuatro instituciones del Sistema de Aseguramiento de la Calidad de la Educación que creó la Ley No 20.529 en el año 2011. Si bien estos estándares no son vinculantes, ni están asociados a sanciones por incumplimiento, la ACE realiza una "Evaluación Indicativa del Desempeño" a todos los establecimientos que reciben subvención del Estado (93\% de los establecimientos escolares del país), con la cual organiza todo su sistema de apoyo y acompañamiento para que estos puedan avanzar en la mejora del establecimiento. 
Mediante un análisis deductivo y de categorías prestablecidas, se interpeló el corpus textual de los documentos seleccionados para dar cuenta de los objetivos del análisis crítico documental. Para Montero y Hochman (2005) ello implica dejar al descubierto el orden de las ideas de un texto, para así visibilizar los argumentos que apoyan estas ideas. Se interrogaron las leyes y los documentos buscando responder a las preguntas: ¿Qué tareas y/o funciones tiene el director? ¿A cuál o cuáles principios del NMP se asocian los documentos analizados? Luego, estas respuestas fueron codificadas con el rol del director al que apuntaba cada cita, para finalmente analizar de manera longitudinal qué rol o roles se han configurado los últimos 40 años, y qué implicancias tiene ello para la escuela donde trabaja. El procedimiento contempló las siguientes etapas:

- Definición de la pregunta de investigación.

- Especificación de los criterios de inclusión/exclusión.

- Formulación de plan de búsqueda.

- Interpretación y presentación de los resultados.

Finalmente, en múltiples y variadas reuniones con el equipo de investigación a cargo, se diseñó un cuadro de citas, donde se cruzó la información entre los principios del NMP y las citas clave de la documentación examinada.

\section{Resultados}

El análisis documental arrojó, en primer lugar, que la totalidad de los principios del NMP están presentes en los documentos analizados y que, por lo tanto, son vinculantes con la política educativa dirigida a directores de escuelas chilenas. La Tabla 2 ilustra el análisis documental realizado. Sin embargo, con fines pragmáticos y de economía de escritura, la exposición de resultados se centra en cuatro de los siete principios. El criterio utilizado para tomar esta decisión fue, principalmente, la densidad de citas asociadas. Los principios seleccionados fueron los siguientes:

- Favorecer instrumentos y estilos de gestión del sector privado;

- instalar normas y medidas de desempeño explícitas; 
- instalar indicadores de resultados más que de procesos; y

- hacer hincapié en la competencia como un incentivo para el logro.

Un resultado emergente que también se desarrolla en esta investigación es el viraje que ha hecho el rol del director hacia el liderazgo pedagógico, transformación que empieza a ser evidente en los documentos normativos de los últimos años, tal como se observa en la última fila de la Tabla 2.

Tabla 2

Sintesis del análisis documental realizado

\begin{tabular}{|c|c|c|c|}
\hline Principios NMP & $\begin{array}{c}\mathrm{N}^{0} \text { de citas } \\
\text { asociadas }\end{array}$ & Cita ejemplificadora & Rol \\
\hline $\begin{array}{l}\text { Favorecer instrumentos } \\
\text { y estilos de gestión del } \\
\text { sector privado: favorecer la } \\
\text { profesionalización. }\end{array}$ & 11 & $\begin{array}{l}\text { [Los directores del sector municipal deben]: } \\
\text { "organizar, supervisar y evaluar el trabajo de } \\
\text { los docentes y del personal regido por la Ley } \\
\mathrm{N}^{\circ} 19.464 \text { " (Ley N } \mathrm{N}^{\circ} 20.501 \text {, art. } 1^{\circ} \text { ). }\end{array}$ & $\begin{array}{l}\text { Director } \\
\text { gerente. }\end{array}$ \\
\hline $\begin{array}{l}\text { Instalar normas y medidas de } \\
\text { desempeño explícitas. }\end{array}$ & 20 & $\begin{array}{l}\text { "Al término del segundo semestre de cada } \\
\text { año escolar y antes del inicio del próximo año } \\
\text { escolar, los directores de los establecimientos } \\
\text { educacionales subvencionados deberán presentar } \\
\text { a la comunidad escolar y a sus organizaciones } \\
\text { un informe escrito de la gestión educativa del } \\
\text { establecimiento correspondiente a ese mismo } \\
\text { año escolar. Tal informe deberá versar sobre, a } \\
\text { lo menos, lo siguiente: a) Las metas y resultados } \\
\text { de aprendizaje del período, fijados al inicio } \\
\text { del año escolar. b) Los avances y dificultades } \\
\text { en las estrategias desarrolladas para mejorar } \\
\text { los resultados de aprendizaje. c) Las horas } \\
\text { realizadas del plan de estudios y el cumplimiento } \\
\text { del calendario escolar. d) Los indicadores de } \\
\text { eficiencia interna: matrícula, asistencia, aprobados, } \\
\text { reprobados y retirados. e) El uso de los recursos } \\
\text { financieros que perciban, administren y que les } \\
\text { sean delegados. f) La situación de la infraestructura } \\
\text { del establecimiento. g) La cuenta deberá incluir } \\
\text { también una relación respecto de las líneas de } \\
\text { acción y compromisos futuros. h) En el caso de } \\
\text { los establecimientos municipales, estos deberán } \\
\text { dar cuenta de los compromisos asumidos en el } \\
\text { PADEM" (Ley No 19.979, art. 11). }\end{array}$ & $\begin{array}{l}\text { Director } \\
\text { gerente. }\end{array}$ \\
\hline $\begin{array}{l}\text { Instalar indicadores de } \\
\text { resultados más que de } \\
\text { procesos. }\end{array}$ & 5 & $\begin{array}{l}\text { "Traducir los propósitos y objetivos institucionales } \\
\text { en planes de mejoramiento y metas de corto y } \\
\text { mediano plazo" (Mineduc, 2015, p. 23). }\end{array}$ & $\begin{array}{l}\text { Director } \\
\text { gerente. }\end{array}$ \\
\hline $\begin{array}{l}\text { Desagregar y descentralizar } \\
\text { los servicios públicos, } \\
\text { entendiendo la desagregación } \\
\text { como transformación de las } \\
\text { organizaciones burocráticas } \\
\text { tradicionales en agencias } \\
\text { independientes. }\end{array}$ & 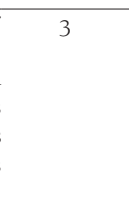 & $\begin{array}{l}\text { "Créase la Agencia de Calidad de la Educación, } \\
\text { en adelante 'la Agencia', servicio público } \\
\text { funcionalmente descentralizado, dotado de } \\
\text { personalidad jurídica y patrimonio propio y que } \\
\text { se relacionará con el Presidente de la República } \\
\text { por intermedio del Ministerio de Educación" (Ley } \\
\text { No } 20.529 \text {, art. 9). }\end{array}$ & $\begin{array}{l}\text { Director } \\
\text { gerente. }\end{array}$ \\
\hline
\end{tabular}




\begin{tabular}{|c|c|c|c|}
\hline $\begin{array}{l}\text { Hacer hincapié en la } \\
\text { competencia como un } \\
\text { incentivo para el logro. }\end{array}$ & 5 & $\begin{array}{l}\text { "La Agencia de la Calidad de la Educación efectuará } \\
\text { la ordenación de estos establecimientos de acuerdo } \\
\text { a los resultados de aprendizaje de los alumnos, } \\
\text { en función del grado de cumplimiento de los } \\
\text { estándares de aprendizaje, referidos a los objetivos } \\
\text { generales señalados en la ley y sus respectivas bases } \\
\text { curriculares, y al grado de cumplimiento de los } \\
\text { otros indicadores de calidad educativa propuestos } \\
\text { por el Ministerio de Educación y aprobados por el } \\
\text { Consejo Nacional de Educación" (Ley N } N^{\circ} 20.529 \text {, } \\
\text { art. 22). }\end{array}$ & $\begin{array}{l}\text { Director } \\
\text { gerente. }\end{array}$ \\
\hline $\begin{array}{l}\text { Introducir contratos } \\
\text { a plazo fijo, pago en } \\
\text { función del rendimiento } \\
\text { y la determinación local } \\
\text { de sueldos y condiciones } \\
\text { laborales. }\end{array}$ & 2 & $\begin{array}{l}\text { "Suscribirán con el respectivo sostenedor un } \\
\text { convenio de desempeño. Este convenio será } \\
\text { público y en él se incluirán las metas anuales } \\
\text { estratégicas de desempeño del cargo durante el } \\
\text { período y los objetivos de resultados a alcanzar por } \\
\text { el director anualmente, con los correspondientes } \\
\text { indicadores, medios de verificación así como } \\
\text { las consecuencias de su cumplimiento e } \\
\text { incumplimiento" (Ley N } \mathrm{N}^{\circ} 20.501 \text {, art. 33). }\end{array}$ & $\begin{array}{l}\text { Director } \\
\text { gerente. }\end{array}$ \\
\hline $\begin{array}{l}\text { Reducción de costos, } \\
\text { limitación de los } \\
\text { presupuestos y mayor } \\
\text { transparencia en la asignación } \\
\text { de recursos. }\end{array}$ & 4 & $\begin{array}{l}\text { "Cada rendición deberá llevar la firma del director } \\
\text { del establecimiento educacional correspondiente, } \\
\text { mediante la cual se confirmará el visto bueno } \\
\text { de este frente a lo presentado por el sostenedor, } \\
\text { previo conocimiento del consejo escolar" (Ley } \\
\mathrm{N}^{0} 20.567 \text {, artículo único). }\end{array}$ & $\begin{array}{l}\text { Director } \\
\text { gerente. }\end{array}$ \\
\hline $\begin{array}{l}\text { Fuera de los principios } \\
\text { del NMP: un giro hacia la } \\
\text { colaboración y el desarrollo } \\
\text { profesional. }\end{array}$ & 10 & $\begin{array}{l}\text { [Los equipos directivos]: "crean una cultura } \\
\text { de colaboración y aprendizaje mutuo entre los } \\
\text { profesionales del establecimiento, para lo cual } \\
\text { generan condiciones necesarias e instancias } \\
\text { sistemáticas, en tiempos no lectivos, de reflexión } \\
\text { y trabajo técnico colectivo sobre la enseñanza y el } \\
\text { aprendizaje de sus estudiantes" (Mineduc, 2015, } \\
\text { p. 23). }\end{array}$ & $\begin{array}{l}\text { Líder } \\
\text { pedagógico. }\end{array}$ \\
\hline
\end{tabular}

Fuente: Elaboración propia.

\section{Favorecer instrumentos y estilos de gestión del sector privado}

Los documentos analizados presentan un discurso instalado propio del sector privado, que busca la profesionalización de los actores de la comunidad escolar, entre ellos, el director de escuela. Esto se logra a través de la aplicación de instrumentos, fiscalización y vigilancia, cuya base está en una forma de gestión que busca un sujeto productivo. De la revisión realizada, se levantan dos áreas que son propias del sector privado y que, en el marco del NMP, se espera que el director de escuela maneje: la gestión de recursos financieros y la gestión de recursos humanos.

Otorgar comprobantes de pago debidamente autorizados por los secretarios regionales ministeriales respectivos por aquellos 
ingresos que perciban, con exclusión de la subvención escolar. Estos ingresos deberán registrarse en libros de contabilidad de acuerdo con las normas de procedimientos usualmente aceptadas y los cuales deberán autorizarse previamente por la Secretaría Regional Ministerial respectiva (Decreto No 8.144, art. 22).

En esta ley de 1980 se menciona por primera vez el uso de libros de contabilidad, algo que vuelve a aparecer en el año 2004:

Llevar contabilidad presupuestaria simplificada, atenerse a las normas sobre administración financiera del Estado contenidas en el Decreto Ley No 1.263, de 1975, a las instrucciones específicas que imparta la Dirección de Presupuestos (Ley No 19.979, art. 24).

De esta manera, se va perfilando un director que debe tener habilidades de gestión financiera, lo que quedará consagrado en la Ley No 19.979 del año 2004, y en el Decreto No 177 del año 2006, donde se explicita que es función del director gestionar administrativa y financieramente el establecimiento. En los años siguientes, se agrega a esta función mantener siempre disponible el balance del año anterior para las visitas de la Superintendencia de Educación (Superintendencia de Educación Escolar, 2013), así como la elaboración de presupuestos y el control de gastos, de tal manera de asegurar la sustentabilidad de la institución (ACE, 2014).

Por otra parte, el nuevo siglo trajo aparejadas nuevas atribuciones en el ámbito de la gestión de recursos humanos al menos en tres aspectos:

1. Definir cargos y funciones: definir los cargos y funciones del personal, los que cumplen con los requisitos estipulados para obtener y mantener el reconocimiento oficial (ACE, 2014).

2. Implementar un sistema de evaluación y retroalimentación del desempeño del personal:

- Organizar y supervisar el trabajo de los docentes y del personal regido por la Ley $\mathrm{N}^{\circ} 19.464$ (Ley No 19.979, art. $7^{\circ}$ bis).

- Organizar, supervisar y evaluar el trabajo de los docentes y del personal regido por la Ley $N^{o} 19.464$ (Ley No 20.501, art. $1^{\circ}$ ). 
- Implementar un sistema de evaluación y retroalimentación del desempeño del personal (ACE, 2014).

3. Despedir profesores: proponer anualmente al sostenedor el término de la relación laboral de hasta un 5\% de los docentes del respectivo establecimiento, siempre que hubieren resultado mal evaluados según lo establecido en el artículo 70 de esta ley (Ley $\mathrm{N}^{\circ} 20.501$, art. $1^{\circ}$ ).

Es interesante el cambio que se observa del año 2004, cuando se especifica que un aspecto de la labor del director es "organizar y supervisar" el trabajo de los docentes, al año 2011 donde, además de esa función, se agrega "evaluar", lo que da pie a otro artículo de la misma ley donde se le otorga la facultad de poner a disposición del sostenedor (desvincular de la escuela) al 5\% de los profesores mal evaluados (según la evaluación nacional docente).

Esta multiplicidad de funciones en el ámbito administrativo/ financiero se entiende como parte del rol del director, que debe liderar y sacar adelante a una escuela o liceo. Teniendo claro el rol, es preciso asegurar en el puesto a los mejores. En esta materia, se sigue otro estilo de gestión del sector privado, ya que el proceso de selección del cargo es externo y transparente, lo cual se piensa que puede asegurar la selección de los mejores directores. El primer paso en este sentido se dio el año 2005, donde la Ley No 20.006 puso fin a los directores vitalicios, estableciendo la obligatoriedad de la concursabilidad del cargo. No fue hasta el año 2011 cuando se definió con mayor rigurosidad el proceso de postulación al cargo a través del Servicio Civil (vigente hasta la fecha):

La selección será un proceso técnico de evaluación de los candidatos que incluirá, entre otros aspectos, la verificación de los requisitos solicitados en el perfil definido en el artículo anterior, entrevistas a los candidatos y la evaluación de los factores de mérito, de liderazgo y de las competencias específicas, cuya ponderación será determinada por cada sostenedor. El proceso de evaluación deberá considerar el apoyo de asesorías externas registradas en la Dirección Nacional del Servicio Civil, con la 
finalidad de preseleccionar los candidatos que serán entrevistados por la comisión calificadora. Estas asesorías deberán ser elegidas por el miembro de la comisión calificadora del Consejo de Alta Dirección Pública, creado en la Ley $N^{\circ} 19.882$, o su representante (Ley $\mathrm{N}^{\circ} 20.501$, art. 32 bis).

No solo el director empieza a configurarse como un profesional que posee un estilo de gestión propio del ámbito privado, sino que las condiciones en las que se desempeña también presentan idéntica característica. Al año 2011, elementos como la evaluación constante, la inspección y la rendición de cuentas van a configurar un contexto laboral que se articula claramente con los principios del NMP. A la vez, estos interpelan directamente al director, elevándolo como una figura capaz de desenvolverse en este estilo de gestión.

El Sistema comprenderá, entre otros, procesos de autoevaluación, evaluación externa, inspección, pruebas externas de carácter censal y, cuando corresponda, apoyo técnico pedagógico en la elaboración e implementación de planes de mejora educativa a nivel de establecimientos que permitan desarrollar sus fortalezas y superar sus debilidades. El Sistema contemplará, además, la rendición de cuentas de los diversos actores e instituciones del sistema escolar y, en particular, de los establecimientos educacionales (Ley No 20.529, art. $2^{\circ}$ ).

\section{Normas y medidas de desempeño más explícitas}

En este nuevo escenario, las normas y las medidas de desempeño cobran especial relevancia para configurar y mantener este nuevo estilo de gestión. De esta manera, la ley exige que cuando el director de escuela asume el cargo, debe firmar un convenio con su jefe (sostenedor), en el cual se compromete con metas de desempeño específicas para los cinco años que estará en el cargo:

Suscribirán con el respectivo sostenedor o con el representante legal de la respectiva Corporación Municipal un convenio de desempeño. Este convenio será público y en él se incluirán las metas anuales estratégicas de desempeño del cargo durante el 
período y los objetivos de resultados a alcanzar por el director anualmente, con los correspondientes indicadores, medios de verificación y supuestos básicos en que se basa el cumplimiento de los mismos así como las consecuencias de su cumplimiento e incumplimiento (Ley No 20.501, art. 33).

Además del compromiso firmado con el sostenedor al momento de asumir el cargo, el director se compromete junto con la escuela, a desarrollar un conjunto de acciones destinadas a mejorarla. Estas acciones contienen metas, plazos y responsables, los que quedan consagrados en el Plan de Mejoramiento Educativo (PME). La ley que da origen al PME es la No 20.248 del año 2008, donde se indica que es la "comunidad del establecimiento escolar" la que elabora el PME (Ley $N^{\circ} 20.248$, art. $7^{\circ}$, letra d). Sin embargo, el año 2011 se modifica esta ley, insertando la figura del director en el proceso de elaboración:

Cumplir un Plan de Mejoramiento Educativo elaborado con el director del establecimiento y el resto de la comunidad que contemple acciones (Ley No 20.550, artículo único).

El plan de mejoramiento debe definir metas concretas, prioridades, responsables, plazos y presupuestos. Se debe contar con un sistema efectivo para monitorear el cumplimiento del plan de mejoramiento (ACE, 2014).

El director, fuera de su rol vinculante con los procesos de mejora, debe dar cuenta del logro de objetivos. En la Ley No 19.410, que modifica el estatuto docente, se hace mención a la acción de dar cuenta de los resultados alcanzados, lo que se reafirma con el DFL $\mathrm{N}^{\mathrm{o}} 2$, donde se exige entregar anualmente a los padres y apoderados un informe de la gestión del establecimiento. Esto se consagra el año 2004:

Al término del segundo semestre de cada año escolar (...) los Directores (...) deberán presentar a la comunidad escolar y a sus organizaciones un informe escrito (...). Tal informe deberá versar sobre, a lo menos, lo siguiente: a) Las metas y resultados de aprendizaje del período, fijados al inicio del año escolar. 
b) Los avances y dificultades en las estrategias desarrolladas para mejorar los resultados de aprendizaje. c) Las horas realizadas del plan de estudios y el cumplimiento del calendario escolar. d) Los indicadores de eficiencia interna: matrícula, asistencia, aprobados, reprobados y retirados. e) El uso de los recursos financieros que perciban, administren y que les sean delegados. f) La situación de la infraestructura del establecimiento. g) La cuenta deberá incluir también una relación respecto a líneas de acción y compromisos futuros. h) En el caso de los establecimientos municipales deberán dar cuenta de los compromisos asumidos en el PADEM (Ley No 19.979, art. 11).

\section{El valor está puesto en indicadores de resultados más que de procesos}

En este ejercicio de profesionalización y cumplimiento de normas — ambas ligadas a la producción — resulta predecible pensar un ambiente laboral más vinculante con los resultados obtenidos que con los procesos desarrollados. Las diversas políticas revisadas empiezan a instalar un discurso que busca comprometer a la escuela y a su director en el logro de resultados.

Traducir los propósitos y objetivos institucionales en planes de mejoramiento y metas de corto y mediano plazo, en el marco de procesos de planificación participativos (Mineduc, 2015).

Además, los resultados obtenidos tendrán especial relación con la asignación de dineros y con la clasificación de establecimientos escolares.

Para determinar la clasificación de los establecimientos se deberán considerar los resultados de aprendizaje de sus alumnos, medidos a través de los instrumentos diseñados por el Ministerio de Educación para tal efecto. El número de mediciones en las cuales se muestren dichos resultados en ningún caso podrá ser inferior a dos (Ley 20.248, art. 66). 


\section{Hacer hincapié en la competencia como un incentivo para el logro}

Siguiendo la lógica de la rendición de cuentas y de una gestión más ligada al logro de resultados, la competencia aparece como un elemento constitutivo del trabajo que hace y se hace en las escuelas. Esta competencia configura las formas de operar que tiene actualmente el sistema escolar y que, basadas en las mismas organizaciones escolares, se instalan en el discurso y en las prácticas de la escuela. Una manera de entender esta competencia es ordenar los establecimientos según su rendimiento escolar, que establece categorías para cada uno de ellos:

La ordenación de estos establecimientos la efectuará la Agencia de la Calidad de la Educación de acuerdo a los resultados de aprendizaje de los alumnos, en función del grado de cumplimiento de los estándares de aprendizaje, referidos a los objetivos generales señalados en la ley y sus respectivas bases curriculares, y al grado de cumplimiento de los otros indicadores de calidad educativa propuestos por el Ministerio de Educación y aprobados por el Consejo Nacional de Educación (Ley No 20.529, art. 112).

En el caso de aquellos establecimientos educacionales que no exhiban una mejora significativa luego de tres años de haber sido ordenados como de Desempeño Insuficiente, la Agencia deberá informar a los padres y apoderados de dichos establecimientos educacionales sobre la situación en que estos se encuentran (Ley $\mathrm{N}^{\circ} 20.529$, art. 30).

Es relevante mencionar que en un discurso del Presidente de la República, Sebastián Piñera (Piñera, 2011) deja de manifiesto que el propósito de este sistema de información pública, no es otro que incentivar la libre competencia, donde los padres pueden elegir a qué escuelas ir:

Y por eso nosotros queremos ayudar a los padres, para que los padres ayuden a sus hijos. Y les vamos a entregar información en forma simple, clara, permanente, de cómo le está yendo a sus 
hijos, cómo es la calidad de la escuela donde están sus niños, qué otras escuelas existen en la comuna o en el barrio (p. 3).

\section{Fuera de los principios del NMP: un giro hacia la colaboración y el desarrollo profesional}

Al finalizar el recorrido histórico por las políticas que afectan a los directores de escuelas chilenas, es preciso nombrar a tres que, en lo referido al trabajo del director, están fuera de los principios del NMP y apuntan más bien a un rol de líder pedagógico explícito.

En el año 2015 surgió un nuevo Marco para la buena dirección y liderazgo escolar (MBDLE), que es una actualización del Marco para la buena dirección (MBD) desarrollado 10 años antes. Este nuevo documento presenta tres diferencias respecto de su homónimo del año 2005. En primer lugar, no hace mención a la evaluación de directores, a diferencia del marco del año 2005 donde este es el objetivo principal. En segundo lugar, el nuevo marco está dirigido "a todos los líderes escolares, independiente de la función directiva específica que estos ocupen y no remite exclusivamente al director o directora de un establecimiento escolar, sino al conjunto de docentes que ejercen roles de liderazgo en dicha escuela" (2015, p. 18). Por último, el foco principal de este nuevo marco está relacionado con el liderazgo pedagógico, cuyo objetivo no es otro que apoyar el trabajo de los profesores, fortaleciendo sus capacidades, condiciones de trabajo y motivación (Leithwood et al., 2006; Robinson, Lloyd \& Rowe, 2008). En este sentido, el nuevo MBDLE se aparta de los principios del NMP, relevando el trabajo con otros, la responsabilización colectiva, el desarrollo profesional y el trabajo con profesores en el largo plazo, como claves de la mejora escolar.

En el año 2016 se dicta la Ley No 20.903 que crea el Sistema de Desarrollo Profesional Docente. La literatura especializada en desarrollo profesional docente revela que cuando existen profesores que trabajan de manera colaborativa y reflexiva en la mejora de sus prácticas, aprendiendo unos de otros, ocurren cambios sustantivos en la cultura escolar (Ávalos, 2011). En este sentido, resulta particularmente interesante la relevancia que toma el rol del director 
de escuela en esta materia, donde la ley le demanda "desarrollar las competencias profesionales de los equipos docentes" (Ley No 20.903, art. $1^{\circ}$ ), para lo cual contempla promover:

El trabajo colaborativo entre profesionales de la educación, tendiente a constituir comunidades de aprendizaje, guiadas por directivos que ejercen un liderazgo pedagógico y facilitan el diálogo, la reflexión colectiva y la creación de ambientes de trabajo que contribuyen a mejorar los procesos de enseñanza-aprendizaje (Ley No 20.903, art. 19).

Finalmente, en el año 2017, con la ley que crea el nuevo Sistema de Educación Pública, por primera vez se indica explícitamente en la legislación que el director debe "ejercer el liderazgo técnicopedagógico en el establecimiento a su cargo" (Ley No 21.040, art. 9). Esto contempla que, en conjunto con su equipo, debe organizar, planificar, supervisar, coordinar y evaluar la enseñanza y el aprendizaje de los estudiantes, así como orientar el desarrollo profesional continuo de docentes y asistentes de la educación (Ley $\mathrm{N}^{\circ} 21.040$ ).

Los datos acá analizados revelan que durante los últimos 40 años se han implementado políticas educativas que se sustentan en los distintos principios del NMP. Así también, durante los últimos años, la normativa ha realizado un giro respecto de lo que espera del director, alejándose de lo que consagraban las anteriores políticas managerialistas. En la Figura 1, se presenta una gráfica que permite "mapear" las políticas educativas dirigidas a directores de escuelas chilenas durante los últimos 40 años. En el eje central se encuentran los años en que se proclaman las políticas; por sobre estos, las leyes proclamadas (en cajas rojas); y bajo los años, el elemento del NMP al que están asociadas dichas leyes (en cajas azules). 


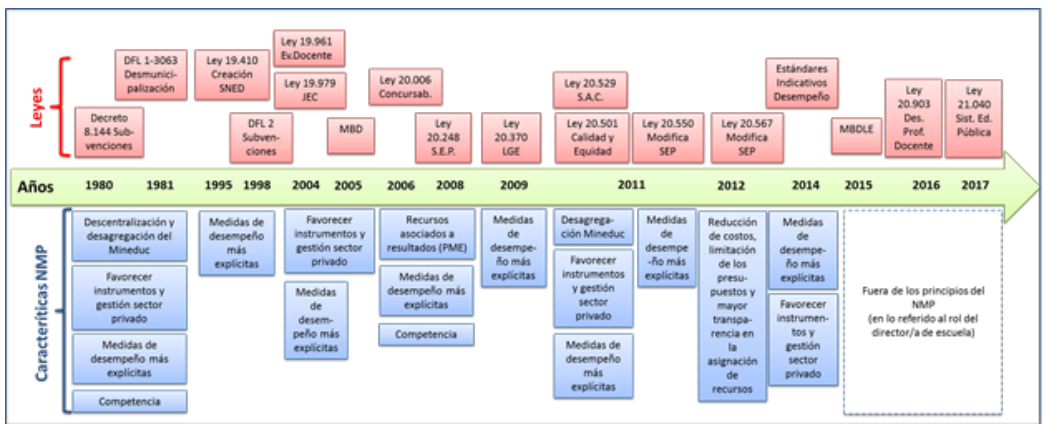

Figura 1. Mapa de políticas implementadas entre 1980 y 2017 que afectan directa 0 indirectamente a directores de escuela y su vinculación con los principios del NMP.

Fuente: Elaboración propia.

\section{Discusión y conclusiones}

La revisión de los documentos realizada en este estudio da cuenta de la alta complejidad que conlleva ser director de escuela. No solo por las múltiples tareas que le demanda la política, sino porque estas, a lo largo de 40 años, han configurado en el papel un doble rol. Por un lado, las políticas le solicitan al director elevar indicadores de efectividad de la escuela (resultados de pruebas estandarizadas, matrícula, asistencia, retención, etc.), y que se evalúe a los profesores, despidiendo a aquellos con bajo desempeño. Esta línea de acción nos habla de un rol "gerencial" del director, donde su propia performatividad sirve como medida de productividad y de calidad de la escuela que dirige (Ball, 2003). Por otro lado, en los últimos años algunos documentos normativos le piden focalizarse en apoyar el trabajo de sus profesores, poniendo énfasis en su desarrollo profesional para la mejora de los aprendizajes de los estudiantes. De esta manera, se va configurando el rol de líder pedagógico, el cual se erige como la esencia misma del rol del director, refrendado por un contundente compendio de investigaciones nacionales e internacionales que lo posicionan como el segundo factor más importante dentro de la escuela después del profesor (Day et al., 2010; Leithwood et al., 2006; Sammons, Gu, Day \& Ko, 2011; Valenzuela y Horn, 2012). 
Ahora bien, al revisar las políticas, pareciese que las tareas del director están claramente definidas, sin embargo, lo que en ellas aparece son más bien los resultados esperados de su desempeño: se espera que el director ejerza un liderazgo pedagógico orientado a trabajar con los profesores en la mejora de sus prácticas pedagógicas, pero también que cumpla con los indicadores comprometidos de matrícula, pruebas estandarizadas, que disponga todo lo que la Superintendencia de Educación necesita cuando fiscalice la escuela, entre otros, con las consecuencias que su incumplimiento puede traer para su persona. Lo que las leyes no dicen es cómo lograr estos objetivos y resolver esta posible tensión entre ambos roles.

Al verlas en perspectiva histórica, se observa que sin importar el color político, estas reformas avanzan año a año: desde su génesis en el gobierno militar, con la salida del Estado de las escuelas y liceos públicos, hasta un gobierno de derecha (2010-2013; 20172021), pasando por gobiernos demócrata cristianos (1990-1999), y socialistas (2000-2009; 2014-2017). Si bien, durante los últimos años existen documentos normativos que, en lo referido al rol del director, se alejan de los principios del NMP, propiciando un liderazgo pedagógico, el problema es que estos conviven en el día a día de la escuela con las otras normas analizadas, provocando una aparente tensión entre ambos roles, cuestión que sería interesante explorar en terreno en un nuevo estudio. De esta manera, la implementación de las políticas bajo el NMP se ejecuta en un continuo ininterrumpido hasta el año 2015: nacen bajo la dictadura respaldada por la derecha, y es seguida por los gobiernos democráticos posteriores, los que no hicieron otra cosa que complementar, guiar y perfeccionar el modelo de mercado heredado de ella (Bellei, 2015); aún más, se incrementaron en la última década. La implementación del NMP de manera transversal a las distintas fuerzas políticas es una situación que Chile comparte con otros países del mundo (Hall \& Gunter, 2015; Klitgaard, 2007; Verger \& Curran, 2015; Verger y Normand, 2015; Wiborg, 2013).

Ahora bien, estas políticas amparadas en el NMP han colaborado en construir, durante los últimos años, el imaginario del director como el profesional que podrá liderar la mejora de la 
educación en cada escuela. De esta forma, se mantiene el mismo fin de mejorar la calidad de la educación, sin embargo, todas las "innovaciones" que se implementan para resolver el problema de la educación, van agregando nuevas tareas y demandas al director de escuela, complejizando su rol. Todo pareciera resolverse proporcionando las condiciones necesarias para que el director haga su trabajo: mayores atribuciones, mayor desarrollo de competencias de liderazgo, mejor reclutamiento y selección (Ley $N^{0} 20.501$ ) y, tal como señaló el Presidente de la República de la época, Sebastián Piñera, "mayor vigilancia y supervigilancia" (Biblioteca del Congreso Nacional, 2011) sobre lo que hace (o no hace) en la escuela. El conjunto de estas evidencias permite ver el "gran" discurso (Ball, 1993) detrás de las políticas, que no es otra cosa que la trampa de una ilusoria "mayor libertad" que tendrían los directores para liderar sus escuelas, eligiendo a su equipo, desarrollando sus capacidades, despidiendo a profesores mal evaluados, manejando mayores recursos y accediendo a más desarrollo profesional. Hablamos de una trampa de ilusoria libertad, dado que el modelo triunfa al alejar al Estado cada vez más y dejar solo al director como único responsable de la mejora de la escuela.

En este escenario, si bien es positivo que durante los últimos años existan normas que se apartan de los principios del NMP, estos documentos no resuelven la tensión entre ambos roles. Resulta irreal pensar en la implementación del liderazgo pedagógico que se argumenta en esos documentos, dado que este sienta sus bases en el trabajo colaborativo y de apoyo al desarrollo profesional de los docentes de la escuela, para lo cual se requiere la implementación de diversas acciones en el mediano y largo plazo. Esto no se condice con las exigencias que la política pública ha hecho mayoritariamente al director de escuela en Chile que, como hemos visto, están amparadas en los principios del NMP, los que apuntan a aspectos totalmente distintos: competencia, emprendimiento individual, metas de desempeño medibles en el corto plazo, etc., los cuales configuran el rol del director como un mero implementador de la agenda política managerialista, más que un profesional reflexivo preocupado por los aprendizajes de sus estudiantes (Fink \& Brayman, 2006). De esta manera, se termina imponiendo este rol de director-gerente, cuya 
función primordial es la gestión de una serie de indicadores duros, que son los que realmente darán cuenta de la supuesta calidad de la escuela que dirige.

En función de los hallazgos encontrados, las proyecciones de este estudio radican en hacer investigaciones que permitan conocer cómo el director resuelve en la escuela estas tensiones entre su rol gerencial y su rol de líder pedagógico. Conjuntamente, también es necesario que quienes nos dedicamos a la investigación educativa, colaboremos en la resolución de estas y otras tensiones que emanan del carácter managerialista de las políticas educacionales.

\section{Referencias}

Agencia de Calidad de la Educación, ACE. (2014). Estándares indicativos de desempeño para los establecimientos educacionales y sostenedores. Recuperado de http://archivos.agenciaeducacion.cl/documentosweb/ Estandares_Indicativos_de_Desempeno.pdf

Andrade Oliveira, D. (2015). Nova gestão pública e governos demorácticopopulares: Contradições entre a busca da eficiência e a ampliação do direito á educação. Educação y Sociedade, 36(132), 625-646. https:// doi.org/10.1590/es0101-73302015152440

Aoki, N. (2015). Institutionalization of new public management: The case of Singapore's education system. Public Management Review, 17(2), 165186. https://doi.org/10.1080/14719037.2013.792381

Ávalos, B. (2011). Teacher professional development in teaching and teacher education over ten years. Teaching and Teacher Education, 27(1), 10-20. https://doi.org/10.1016/j.tate.2010.08.007

Ball, S. J. (1993). What Is policy? Texts, trajectories and toolboxes. Discourse: Studies in the Cultural Politics of Education, 13(2), 10-17. https://doi. org/10.1080/0159630930130203

Ball, S. J. (2003). The teacher's soul and the terrors of performativity. Journal of Education Policy, 18(2), 215-228. https://doi.org/10.1080/0268093 022000043065

Ball, S. J., Maguire, M., Braun, A., \& Hoskins, K. (2011). Policy actors: Doing policy work in schools. Discourse: Studies in the Cultural Politics of Education, 32(4), 625-639. https://doi.org/10.1080/01596306.201 1.601565 
Bell, L. \& Stevenson, H. (2006). Education policy: Education policy process, themes and impact. Recuperado de http://eprints.lincoln.ac.uk/1851/1/ Ed_Policy_book_proofs.pdf

Bellei, C. (2015). El gran experimento. Mercado y privatización de la educación chilena. Santiago de Chile: LOM Ediciones.

Bellei, C., Valenzuela, J. P., Vanni, X., y Contreras, D. (2014). Lo aprendí en la escuela ¿Cómo se logran procesos de mejoramiento escolar? Santiago de Chile: LOM Ediciones.

Biblioteca del Congreso Nacional (2011). Historia de la Ley No 20.529. Recuperado de https://www.bcn.cl/historiadelaley/nc/historia-de-laley/4584/

Bonal, X. (2002). Globalización y política educativa: un análisis crítico de la agenda del Banco Mundial para América Latina (Globalization and education policy: A critical analysis of the World Bank's agenda for Latin America). Revista Mexicana de Sociología, 64(3), 3-35. https://doi. org/10.2307/3541389

Bulach, C., Boothe, D., \& Pickett, W. (2006). Analyzing the leadership behavior of school principals. Recuperado de https://cnx.org/contents/7PdrvCoS@1/ Analyzing-the-Leadership-Behav

Day, C., Sammons, P., Hopkins, D., Harris, A., Leithwood, K., Gu, Q., \& Brown, E. (2010). 10 strong claims about successful school leadership. Recuperado de https://www.gov.uk/government/uploads/system/ uploads/attachment_data/file/327938/10-strong-claims-aboutsuccessful-school-leadership.pdf

Decreto No 177 Modifica Decreto No 453, de 1991, del Ministerio de Educación. Diario Oficial de la República de Chile, 20 de abril de 2006. Recuperado de https://www.leychile.cl/Navegar?idNorma=249020

Decreto No 8.144 Reglamenta Decreto Ley No 3.476, de 1980, Sobre Subvenciones a Establecimientos Particulares Gratuitos de Enseñanza, del Ministerio de Educación. Diario Oficial de la República de Chile, 4 de septiembre de 1980. Recuperado de https://www.leychile.cl/ Consulta $/ \mathrm{m} / \mathrm{mail}$ ?idNorma $=19462 \&$ \&org=

Decreto con Fuerza de Ley No 2 Fija Texto Refundido, Coordinado y Sistematizado del Decreto con Fuerza de Ley No 2, de 1996, sobre Subvención del Estado a Establecimientos Educacionales, del Ministerio de Educación. Diario oficial de la República de Chile, 20 de agosto de 1998. Recuperado de https://www.leychile.cl/Consulta/m/ mail?idNorma $=127911$ \&org= 
Derouet, J.-L., Normand, R., y Pacheco, R. G. (2015). La modernización del sistema educativo en Francia: la Nueva Gestión Pública entre la afirmación del Estado y la gobernanza descentralizada. Educação y Sociedade, 36(132), 723-741. https://doi.org/10.1590/es010173302015152583

Falabella, A. (2014). The performing school: The effects of market y accountability policies. Education Policy Analysis Archives, 22(70), 1-29. https://doi.org/10.14507/epaa.v22n70.2014

Fink, D. \& Brayman, C. (2006). School leadership succession and the challenges of change. Educational Administration Quarterly, 42(1), 6289. https://doi.org/10.1177/0013161x05278186

Gewirtz, S. \& Ball, S. J. (2000). From "Welfarism" to "New Managerialism": Shifting discourses of school headship in the education marketplace. Discourse: Studies in the Cultural Politics of Education, 21(3), 253-268. https://doi.org/10.1080/713661162

Grimaldi, E., Serpieri, R., \& Taglietti, D. (2015). Jogos da verdade. A nova gestão pública e a modernização do Sistema Educacional Italiano. Educação y Sociedade, 36(132), 759-778. https://doi.org/10.1590/ es0101-73302015152327

Gurr-Mark, D., Drysdale-George, L., \& Mulford, B. (2010). Australian principal instructional leadership: Direct and indirect influence. Magis. Revista Internacional de Investigación en Educación, 2(4), 299-314.

Hall, D., Grimaldi, E., Gunter, H. M., Moller, J., Serpieri, R., \& Skedsmo, G. (2015). Educational reform and modernisation in Europe: The role of national contexts in mediating the new public management. European Educational Research Journal, 14(6), 487-507. https://doi. org/10.1177/1474904115615357

Hall, D. \& Gunter, H. M. (2015). New public management in England: The permanent instability of neo-liberal reform. Educação y Sociedade, 36(132), 743-758.

Hall, D. \& McGinity, R. (2015). Conceptualizing teacher professional identity in neoliberal times: Resistance, compliance and reform. Education Policy Analysis Archives, 23(88), 1-17. https://doi.org/10.14507/epaa. v23.2092

Hood, C. (1991). A public management for all seasons? Public Administration, 69(1), 3-19. https://doi.org/10.1111/j.1467-9299.1991.tb00779.x

Klitgaard, M. B. (2007). Why are they doing it? Social democracy and marketoriented welfare state reforms. West European Politics, 30(1), 172-194. https://doi.org/10.1080/01402380601019753 
Kogan, M. (1975). Educational policy making. A study of interest groups and parliment. London: Routledge.

Leithwood, K., Day, C., Sammons, P., Harris, A., \& Hopkins, D. (2006). Successful school leadership what it is and how it influences pupil learning. Recuperado de http://illinoisschoolleader.org/research_compendium/ documents/successful_school_leadership.pdf

Lerena, B. y Trejos, J. (2015). Sobre la posibilidad de una educación inclusiva bajo el actual modelo de desarrollo económico y social chileno. Revista Latinoamericana de Educación Inclusiva, 9(2), 145-160.

Ley N ${ }^{\circ} 19.070$ Aprueba Estatuto de los Profesionales de la Educación del Ministerio de Educación. Diario Oficial de la República de Chile, 1 de julio de 1991. Recuperado de https://www.leychile.cl/ Navegar?idNorma $=30437$

Ley No 19.410 Modifica la Ley No 19.070, sobre Estatuto de Profesionales de la Educación, el Decreto con Fuerza de Ley No 5, de 1993, del Ministerio de Educación, Sobre Subvenciones a Establecimientos Educacionales, y Otorga Beneficios que Señala del Ministerio de Educación. Diario Oficial de la República de Chile, 2 de septiembre de 1995. Recuperado de https://www.leychile.cl/Navegar?idNorma=30777

Ley No 19.979 Modifica el Régimen de Jornada Escolar Completa Diurna y Otros Cuerpos Legales, del Ministerio de Educación. Diario Oficial de la República de Chile, 6 de noviembre de 2004. Recuperado de https:// www.leychile.cl/Navegar?idNorma $=232146$

Ley N 20.006 Restablece Concursabilidad de los Cargos de Directores de Establecimientos Educacionales Municipales. Diario oficial de la República de Chile, 22 de marzo de 2005. Recuperado de https://www. leychile.cl/Navegar?idNorma $=236496$

Ley No 20.248 Subvención Escolar Preferencial del Ministerio de Educación. Diario Oficial de la República de Chile, 1 de febrero de 2008. Recuperado de https://www.leychile.cl/Navegar?idNorma=269001

Ley No 20.370 Establece la Ley General de Educación del Ministerio de Educación. Diario Oficial de la República de Chile, 12 de septiembre de 2009. Recuperado de https://www.leychile.cl/Navegar?idNorma= $1006043 \&$ \&idParte $=$

Ley No 20.501 Calidad y Equidad de la Educación. Diario Oficial de la República de Chile, 26 de febrero de 2011. Recuperado de https:// www.leychile.cl/Navegar?idLey=20501

Ley No 20.529 Sistema Nacional de Aseguramiento de la Calidad de la Educación Parvularia, Básica y Media y su Fiscalización del 
Ministerio de Educación. Diario Oficial de la República de Chile, 27 de agosto de 2011. Recuperado de https://www.leychile.cl/ Navegar?idNorma $=1028635$

Ley No 20.550 Modifica Ley No 20.248 SEP. Diario Oficial de la República de Chile, 26 de octubre de 2011. Recuperado de https://www.leychile.cl/ Navegar?idNorma $=1030934$

Ley No 20.567 Modifica la Ley No 20.248 de Subvenciones Escolares, en Materia de Rendición del Ministerio de Educación. Diario oficial de la República de Chile, 02 de febrero de 2012. Recuperado de https://www. leychile.cl/Navegar?idNorma $=1036885$ \&tipoVersion $=0$

Ley No 20.903 Crea el Sistema de Desarrollo Profesional Docente y Modifica otras Normas del Ministerio de Educación. Diario Oficial de la República de Chile, 1 de abril de 2016. Recuperado de https://www.leychile.cl/ Navegar?idNorma $=1087343$

Ley No 21.040 Crea el Sistema de Educación Pública del Ministerio de Educación. Diario oficial de la República de Chile, 24 de noviembre de 2017. Recuperado de https://www.leychile.cl/ Navegar?idNorma=1111237

López-Alfaro, P., Osorio-González, F., Gallegos-Araya, V., y Cáceres-Cadena, M. D. (2016). Liderazgo escolar y eficacia colectiva en escuelas públicas de Bogotá. Magis. Revista Internacional de Investigación en Educación, 9(18), 67-84. https://doi.org/10.11144/javeriana.m9-18.leec

Mandiola, M. \& Ascorra, P. (2010). Chilean management education: rhetoric of pragmatism, consumerism, individualism and elitism. Cadernos EBAPE.BR, 8(2), 371-387. https://doi.org/10.1590/s167939512010000200012

Maroy, C., Mathou, C., Vaillancourt, S., \& Voisin, A. (2015). Nova gestão pública e educação: a trajetória da política do quebec de "gestão orientada por resultados." Educação y Sociedade, 36(132), 801-818. https://doi.org/10.1590/es0101-73302015152427

Ministerio de Educación de Chile, Mineduc. (2005). Marco para la buena dirección. Criterios para el desarrollo profesional y evaluación del desempeño. Recuperado de http://www.cpeip.cl/usuarios/cpeip/File/ Documentos2011/marcobuenadireccion.pdf

Ministerio de Educación de Chile, Mineduc. (2015). Marco para la buena dirección y el liderazgo escolar. Santiago: Maval Ltda.

Møller, J. \& Skedsmo, G. (2013). Modernising education: New public management reform in the Norwegian education system. Journal of Educational Administration and History, 45(4), 336-353. https://doi.or g/10.1080/00220620.2013.822353 
Montecinos, C., Pino, M., Campos-Martínez, J., Domínguez, R., \& Carreño, C. (2014). Master teachers as professional developers: Managing conflicting versions of professionalism. Educational Management Administration and Leadership, 42(2), 275-292. https://doi. org/10.1177/1741143213502191

Montecinos, C., Ahumada, L., Galdames, S., Campos, F., \& Leiva, M. V. (2015). Targets, threats and (dis)trust: The managerial troika for public school principals in Chile. Education Policy Analysis Archives, 23(87), 1-29. https://doi.org/10.14507/epaa.v23.2083

Montero, M. y Hochman, E. (2005). Investigación documental: técnicas y procedimientos. Venezuela: Panapo.

Mundy, K. \& Menashy, F. (2014). The World Bank and private provision of schooling: A look through the lens of sociological theories of organizational hypocrisy. Comparative Education Review, 58(3), 401427. https://doi.org/10.1086/676329

Mundy, K. \& Verger, A. (2015). The World Bank and the global governance of education in a changing world order. International Journal of Educational Development, 40, 9-18. https://doi.org/10.1016/j.ijedudev.2014.11.021

Nuñez, I., Weinstein, J., y Muñoz, G. (2012). ¿Posición olvidada? Una mirada desde la normativa a la historia de la dirección escolar en Chile. En J. Weinstein y G. Muñoz (Eds.), ¿Qué sabemos sobre los directores de escuela en Chile? (pp. 371-396). Santiago de Chile: Salesianos Impresores S.A.

Peña, T. y Pirela, J. (2007). La complejidad del análisis documental. Información, Cultura y Sociedad, 16, 55-81.

Piñera, S. (2011). Discurso de promulgación Ley No 20.529. Recuperado de http://www.bcn.cl/historiadelaley/nc/historia-de-la-ley/4584/

Pollitt, C. (1995). Justification by works or by faith?: Evaluating the new public management. Evaluation, 1(2), 133-154. https://doi. org/10.1177/135638909500100202

Ranson, S. (1995). Theorising education policy. Journal of Education Policy, 10(4), 427-448. https://doi.org/10.1080/0268093950100408

Redondo, J. M. (2005). El experimento chileno en educación: ¿Conduce a mayor equidad y calidad en la educación? Última Década, 13(22), 95110. https://doi.org/10.4067/s0718-22362005000100005

Robinson, V. M. J., Lloyd, C. A., \& Rowe, K. J. (2008). The impact of leadership on student outcomes: An analysis of the differential effects of leadership types. Educational Administration Quarterly, 44(5), 635674. https://doi.org/10.1177/0013161x08321509 
Saavedra, J. y Farías, F. (2014). Construcción neoliberal de la política social chilena en el discurso de Pinochet. Revista Katálysis, 1, 22-30. https:// doi.org/10.1590/s1414-49802014000100003

Samaratunge, R., Alam, Q., \& Teicher, J. (2008). The new public management reforms in Asia: A comparison of South and Southeast Asian countries. International Review of Administrative Sciences, 74(1), 25-46. https://doi. org/10.1177/0020852307085732

Sammons, P., Gu, Q., Day, C., \& Ko, J. (2011). Exploring the impact of school leadership on pupil outcomes: Results from a study of academically improved and effective schools in England. International Journal of Educational Management, 25(1), 83-101. https://doi. org/10.1108/09513541111100134

Sisto, V. (2012). Identidades desafiadas: individualización, managerialismo y trabajo docente en el Chile Actual. Psikhé, 21(2), 35-46. https://doi. org/10.7764/psykhe.21.2.542

Sisto, V. y Fardella, C. (2011). Nuevas políticas públicas, epocalismo e identidad: el caso de las políticas orientadas a los docentes en Chile. Revista de Estudos Universitários. REU, 37(1), 123-141.

Stecher, A. (2010). El análisis crítico del discurso como herramienta de investigación psicosocial del mundo del trabajo. Discusiones desde América Latina. Universitas Psychologica, 9(1), 93-107. https://doi. org/1657-926793

Superintendencia de Educación Escolar (2013). Circular $N^{o} 1$. Establecimientos educacionales subvencionados municipales y rarticulares. Recuperado de http://gobta.supereduc.cl/docs/1_amarillo.pdf

Tseng, C. Y. (2015). Changing headship, changing schools: How management discourse gives rise to the performative professionalism in England (1980s-2010s). Journal of Education Policy, 30(4), 483-499. https:// doi.org/10.1080/02680939.2014.972988

Valenzuela, P. y Horn, A. (2012). Influencia del liderazgo directivo en los resultados de los estudiantes. En José Weinstein y G. Muñoz (Eds.), ¿Qué sabemos sobre los directores de escuela en Chile? (pp. 325-348). Santiago de Chile: Centro de Estudios de Políticas y Prácticas en Educación.

Van der Sluis, M. E., Reezigt, G. J., \& Borghans, L. (2015). Implementing New Public Management in Educational Policy. Educational Policy, 31(3), 303-329. https://doi.org/10.1177/0895904815598393 
Verger, A. \& Curran, M. (2014). New public management as a global education policy: Its adoption and re-contextualization in a Southern European setting. Critical Studies in Education, 55(3), 253-271. https:// doi.org/10.1080/17508487.2014.913531

Verger, A. y Curran, M. (2015). La trayectoria de una reforma educativa global: el caso de la nueva gestión pública en el sistema educativo catalán. Educação y Sociedade, 36(132), 675-697. https://doi.org/10.1590/ es0101-73302015152619

Verger, A. y Normand, R. (2015). Nueva gestión pública y educación: elementos teóricos y conceptuales para el estudio de un modelo de reforma educativa global. Educação y Sociedade, 36(132), 599-622. https://doi.org/10.1590/es0101-73302015152799

Wiborg, S. (2013). Neo-liberalism and universal state education: the cases of Denmark, Norway and Sweden 1980-2011. Comparative Education, 49(4), 407-423. https://doi.org/10.1080/03050068.2012.700436

Wittmann, E. (2008). Align, don't necessarily follow. Educational Management Administration y Leadership, 36(1), 33-54. https://doi. org/10.1177/1741143207084059

World Bank. (1997). World development report 1997: The state in a changing world. New York: Oxford University Press. Recuperado de https:// openknowledge.worldbank.org/handle/10986/5980 